CASE REPORT

\title{
A polymorphic challenge
}

\section{Giuseppe A. Ramirez ${ }^{1}$, Maurilio Ponzoni ${ }^{2}$, Luisa Praderio ${ }^{1}$, Moreno Tresoldi ${ }^{1}$, Valentina Canti ${ }^{1}$, Nicoletta Saporiti ${ }^{1}$, Maria Grazia Sabbadini ${ }^{1}$}

1. Department of Immunology, Istituto Scientifico San Raffaele and Università Vita-Salute San Raffaele, Milano, Italy. 2. Pathology Unit, Istituto Scientifico San Raffaele, Milano, I taly

Correspondence: Giuseppe A. Ramirez. Address: Department of Immunology, Istituto Scientifico San Raffaele and Università Vita-Salute San Raffaele, Milano, Italy. Telephone: 390-226-432-654. E-mail: gronk@fastwebnet.it

Received: May 10, 2012

DOI : $10.5430 /$ jhm.v2n3p38
Accepted: June 24, $2012 \quad$ Published: September 1, 2012

URL: http://dx.doi.org/10.5430/jhm.v2n3p38

\section{Abstract}

We describe the case of a 19-year-old boy, who presented with diffuse lymph node enlargements and prominent involvement of his parotid glands. After a four-month history of unsuccessful diagnostic investigations, he was finally diagnosed with Kikuchi-Fujimoto's disease (KFD), a rare histiocytosis of unknown aetiology affecting lymph-nodes throughout the body. The diagnosis of KFD can only be made by histopathologic characterization of affected lymph-nodes, while, according to literature, clinical and imaging features can only suggest the presence of such particular disease. Furthermore, as happened in the case of our patient, KFD tends to be hardly distinguishable from lymphoid malignancies and to overlap with other systemic immune-mediated diseases, such as adult onset Still's disease and systemic lupus erythematosus. The significant involvement of intra-parotid lymph-nodes in our patient's case further extended the diagnostic spectrum to include Sjögren's syndrome.

\section{Key words}

Kikuchi-Fujimoto's disease, Lupus, Lymph-node enlargement, Lymphoma, Macrophage, Adult onset Still's disease, Sjögren's syndrome

\section{Introduction}

KFD is a rare disease of unknown aetiology affecting lymph-nodes throughout the body ${ }^{[1]}$. Its frequency appears to be higher in Asian countries, in females (an issue likely overestimated), and in patients younger than 40 years ${ }^{[2]}$. The clinical course tends to be self-limited, although fatal cases or persistence of the associated autoimmune diseases are also reported ${ }^{[1,3]}$. KFD can affect virtually any lymph node in the body, while number and site of lymph node involvement can vary significantly among patients, making it difficult to even suspect this kind of disease in early stages ${ }^{[1]}$. The frequent association of lymphadenopathy with fever and weight loss in KFD often misleads the clinician towards a diagnosis of lymphoproliferative disease, which indeed is the main element to be excluded in differential diagnosis ${ }^{[2]}$. Moreover specific sites of lymph node involvement can drive the diagnostic cascade towards specific groups of diseases: for example a selective involvement of the cervical lymph nodes could point towards a diagnosis of Hodgkin's lymphoma or Bartonella infection, while the involvement of intra-parotid lymph-nodes could be associated with the presence of infectious or autoimmune (i.e. Sjögren's syndrome) parotitis ${ }^{[4]}$. Laboratory tests in KFD usually show signs of intense inflammation (with elevation of Erythrocyte Sedimentation Rate, C-reactive protein and ferritin levels) and increased 
cellular turnover (with frequent elevation of transaminase and lactate dehydrogenase levels) ${ }^{[3]}$, thus extending the spectrum of the differential diagnosis towards autoinflammatory diseases like adult onset Still's disease ${ }^{[3]}$. Furthermore frequent and significant serological signs of antinuclear immunity can be frequently detected in KFD, pointing towards a clinical (and possibly pathogenic) overlap of KFD with connective tissue diseases and especially systemic lupus erythematosus ${ }^{[5]}$.

In this article a presentation of a real clinical case will be organized in stages. A brief description of the patient's clinical characteristics (preceded by "D") is followed by clinician's commentaries (preceded by "C"). A final discussion of the whole case is provided at the end of the manuscript.

\section{Case presentation}

D: A 19-year-old obese caucasian male presented to an otorhinolaryngologist (ORL) for a 3-months duration of widespread cervical lymph node swelling and painful bilateral parotid enlargement.

C: A diffuse enlargement of lymph nodes in the neck region at presentation is a very common sign among young adults and it is usually attributed to transient oropharyngeal infections, mostly represented by infectious mononucleosis. Alternative potential infectious agents usually encompass Toxoplasma gondii, Borrelia burgdorferi, atypical Mycobacteria, Brucella species, Bartonella henselae and Human Immunodeficiency Virus. A long lasting lymph node enlargement may raise the suspicion of an underlying lymphoproliferative disorder. When analyzed separately, individual signs/symptoms may suggest certain diseases. For instance, a painful enlargement of the parotid glands is commonly associated to mumps, which can require long remission times. A mucous secretion should be expected during squeezing of the parotid in such case. The association of lymph node swelling and parotid inflammation may suggest a diagnosis of Sjögren's syndrome, a disease relatively frequent in early adulthood (although mostly in females) ${ }^{[6]}$, when it shows an intense immunological activation (i.e. marked systemic inflammation and high titres of antinuclear antibodies). The occurrence of a decreased salivary and/or ocular secretion can further support such diagnosis.

D: The patient denied both xerophthalmia and xerostomia. He had regularly been vaccinated for mumps, rubeola, measles, diphtheria, poliomyelitis, tetanus and hepatitis B. During the last months he had been intensively investigated for his parotid and lymph node enlargement but no definite diagnosis was reached: an ultrasonography and a contrast-enhanced magnetic resonance of the neck confirmed the presence of multiple, bilateral enlarged lymph-nodes (diameter range = 0.93-2 cm) with inflammatory features in the sub-mandibular, latero-cervical and supra-clavicular regions, and showed bilateral intra-glandular lymph node enlargements within his swollen parotids. A single 9-mm-large intraparotid lymph node was found to have necrotic appearance. A set of laboratory tests revealed a mild leukopenia (white blood cells count

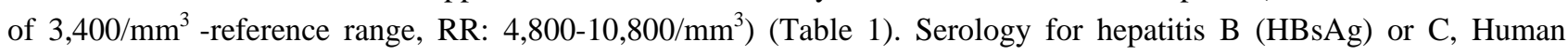
Immunodeficiency Virus, Toxoplasma gondii and Borrelia burgdorferi were negative. Anti Herpes simplex virus 1 and 2 IgG, not IgM, were positive. Epstein Barr virus and Cytomegalovirus DNA assessment by Polymerase Chain Reaction yielded negative results. ORL examination showed a normal tympanic membrane, no morphologic alterations of the nasal and oral cavities and normal appearance of saliva at parotid squeezing.

C: In this case most frequent infective aetiologies have been ruled out, while a primary inflammatory/autoimmune involvement of the salivary glands (i.e. Sjögren's syndrome) appears to fit well to the current clinical scenario, despite the absence of a significant decrease in exocrine secretion (which however occurs less frequently in early onset Sjögren's syndrome ${ }^{[6]}$ ). On the other hand, the presence of lymphoid malignancies has not yet been excluded.

D: In order to confirm the clinical hypothesis of Sjögren's syndrome, the ORL requested anti SS-A and SS-B antibodies. A week after ORL visit, the patient was admitted to an emergency department for the onset of acute bilateral pain in his knees and severe stiffness, which was partially relieved by movement. On physical examination both knees appeared normal, 
and no signs of trauma were identified. A chest and knee X-ray were negative. Laboratory tests confirmed a mild leukopenia and showed a C-reactive protein level of $11.8 \mathrm{mg} / \mathrm{L}$ (RR: 2-6 mg/L) (Table 1). The patient was treated with low dose non-steroidal antinflammatory agents and discharged from the emergency department.

C: Symmetric inflammatory involvement of large joints is a common feature of many diseases, most of which are immune-mediated. Notwithstanding the lack of typical inflammatory signs (i.e., rubor, calor, tumor), the occurence of joint pain and stiffness, relieved by movement, is highly suggestive for arthritis. Moreover the age and the history of the patient rule out most alternative causes of joint pain. The association of arthritis and parotid inflammation further support a diagnosis of Sjögren's syndrome ${ }^{[7]}$, but the presence of lymph node enlargements in association with a systemic inflammatory state could still be consistent with a diagnosis of hematologic malignancy.

Table 1. Summary of the main blood parameters

\begin{tabular}{llll}
\hline & First evaluation by ORL & Emergency Department & At admission \\
\hline White blood cells/mm & 3400 & 4310 & 1900 \\
Haemoglobin (g/dL) & & 12.7 & 10.4 \\
Platelets/mm ${ }^{3}$ & & 120,000 \\
AST (U/L) & & 48 & 175 \\
ALT (U/L) & 72 & & 103 \\
LDH (U/L) & 114 & & 537 \\
GGT (U/L) & 32 & 43 \\
ALP (U/L) & 64 & & 19 \\
ESR (mm/h) & 41 & & 60 \\
C-reactive protein (mg/L) & & 4.9 \\
Na (mmol/L) & & 135 \\
K (mmol/L) & & 3.89 \\
Ferritin (ng/mL) & & 1991 \\
C3 (g/L) & & 0.60 \\
C4 (g/L) & & 0.24 \\
ANA & & $>1: 640$ speckled \\
Anti SS-A & & Positive \\
Anti SS-B & & Negative \\
ADNA & & Positive (1:80) \\
Anti Sm & & Positive \\
Anti RNP & & Positive \\
Lupus Like Anticoagulants & Negative \\
Anti Cardiolipin Antibodies & & Negative \\
\hline
\end{tabular}

Abbreviations: AST: aspartate aminotransferase, ALT: alanine aminotransferase, GGT: gamma glutamil-transferase, ALP: alkaline phosphatase, ESR: erythrocyte sedimentation rate, ANA: anti-nuclear antibodies

D: Few days soonafter the patient reported the occurrence of recurrent nocturnal febrile episodes (body temperature at peak $=39{ }^{\circ} \mathrm{C}$ ). He was given empiric levofloxacin therapy, with no significant improvement. Meanwhile, the search for anti SS-A antibodies resulted positive and thus the patient was referred to our Immunology Department, four months from the onset of his symptoms. On physical examination cardiac rate was 80 /minute, blood pressure $120 / 80 \mathrm{mmHg}$ and oxygen saturation on room air was normal. Chest and abdominal examination were normal. Bilateral swellings of multiple lymph nodes were present in the cervical and inguinal regions. The parotid glands were markedly swollen; shoulders and knees were painful at palpation and limited in flexion because of pain; no sign of joint effusion, hyperthermia or redness were noticed. Ocular and oral secretions appeared normal. Although still obese, the patient reported a decrease of $24 \mathrm{~kg}$ in the last two months. The patient denied any rash, oral ulcer or eye dryness and reported the development of Raynaud's phenomenon 3 months earlier. Laboratory tests showed a mild pancytopenia (white blood cells 1,900/mm ${ }^{3}$-RR:

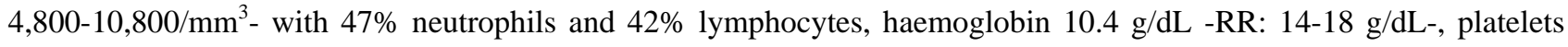




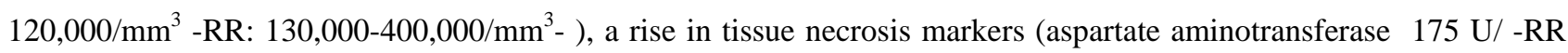
5-41 U/L-, alanine aminotransferase 103 U/L -RR: 6-55U/L-, lactate dehydrogenase 537 U/L -RR: 125-220 U/L-), Erythrocyte Sedimentation Rate $60 \mathrm{~mm} / \mathrm{h}$ (RR: 1-15 mm/h), C-reactive protein $4.9 \mathrm{mg} / \mathrm{L}(\mathrm{RR}: 2-6 \mathrm{mg} / \mathrm{L})$ and ferritin 1,991 ng/mL (RR: 30-400 ng/mL) (Table 1). Serologic tests for HIV, Herpesvirus 6 and 8, Cytomegalovirus, Epstein Barr virus, Hepatitis B virus, Parvovirus, Brucella, Leishmania were negative. Upon admission the patient was still complaining of recurrent fever, despite two weeks of antimicrobial therapy; a new chest X-ray showed again no signs of infection. A chest and abdomen CT scan were performed (see Figure 1), and revealed systemic lymphoadenopathy (mediastinal, axillary, coeliac, perisplenic, lumboaortic, porto-caval, iliac and inguinal regions). A contrast enhanced magnetic resonance of the brain was negative (not shown).

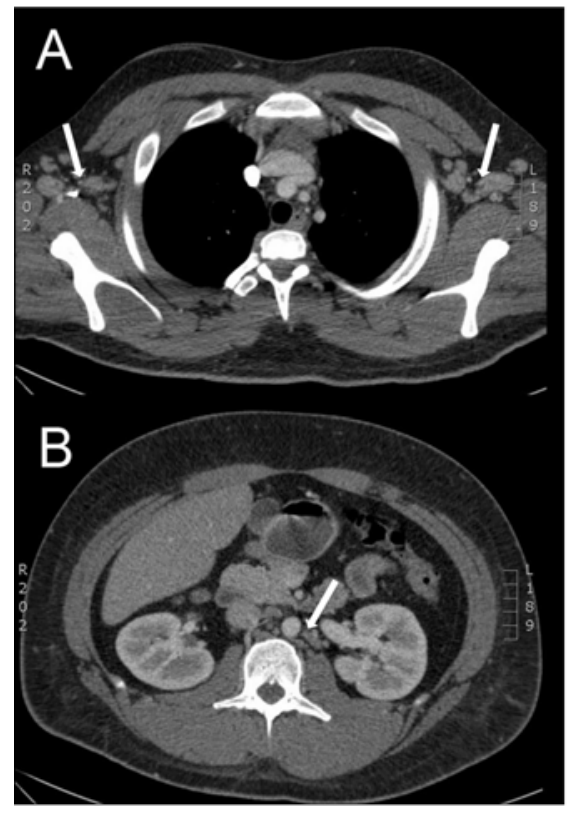

Figure 1. Multiple bilateral lymph-nodes enlargements in the axillary regions (A) and involvement of the lumboaortic lymph-nodes (B).

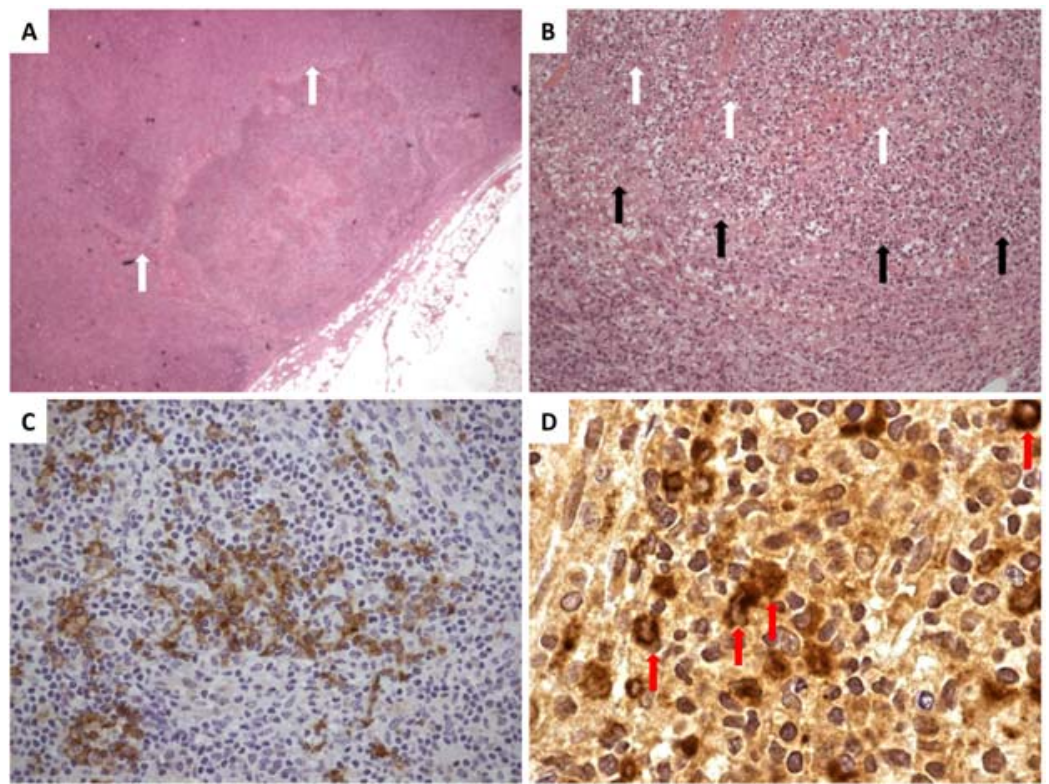

Figure 2. Histologic images of an inguinal lymph-node, showing partial architectural disassembly of the lymph-node due to hyperplasia within the paracortical area (A: white arrows); 20× magnification of the paracortical area showing multiple necrotic foci (white arrows) surrounded by infiltrating cells of monocytic appearance (black arrows) and lack of granulocytes (B); $40 \times$ magnification showing focal aggregates of CD303-positive plasmacytoid dendritic cells ; myeloperoxidase-positive staining of infiltrating monocytes at $100 \times$ magnification (D: red arrows).

C: The presence of the anti SS-A antibodies further supports the earlier hypothesis of Sjögren's syndrome ${ }^{\text {[7]; }}$ however, a cascade of additional events occurred since first ORL examination. Fever is not a classical feature of Sjögren's syndrome and no infectious events have been identified so far. Along with these features, recurrent, non-infectious fever in association with arthritis, elevation of liver enzymes and ferritin could be actually evocative for adult Still's disease. However, no rash has been observed in the clinical history of this patient and the presence of leukopenia strongly argues against such diagnosis.

Mild to severe blood cytopenia is a classical feature of Systemic Lupus Erythematosus (SLE), in which arthritis and Raynaud's phenomenon are also very common. In addition, the typical SLE-associated low C-reactive protein response, when compared to accelerated erythrocyte sedimentation rate ${ }^{[8]}$ (which possibly constitutes a shared behaviour between C-reactive protein and other members of the pentraxin family ${ }^{[9]}$ ), was present in our patient as well. The presence of anti-nuclear antibodies (ANA) is the hallmark of SLE and since this patient has already had anti SS-A, assessment of ANA and other SLE autoantibodies would likely reinforce this diagnostic hypothesis. In addition fever and systemic lymph node 
enlargement are possible clinical presentations of SLE ${ }^{[10]}$ and can be observed in a minority of patients as part of Histiocytic Necrotizing Lymphadenitis, also known as Kikuchi-Fujimoto's Disease (KFD) ${ }^{[3,5]}$. Along this line, a single lymph node of necrotic appearance was noticed early in the course of this patient's history. Despite its frequent association with SLE and other autoimmune diseases, KFD may not be clearly distinguishable from lymphoma without histopathologic analysis. In fact the occurrence of signs of autoimmunity (like autoimmune thrombocytopenia and the presence of autoantibodies) is a clinical feature shared by both autoimmune diseases and many hematologic malignancies including lymphoma.

D: Further investigation of autoimmune serology revealed the presence of ANA at high titer ( $>1: 640$ with speckled pattern). Positivity of the anti DNA (ADNA), anti Sm, anti RNP antibodies and a low complement (C3 and C4) value were also found.

On day 8 an inguinal lymph node was biopsied (see Figure 2). Along with areas of florid follicular hyperplasia, areas of necrosis without neutrophilic infiltration were observed. In the necrotic areas, prominent karyorrhexis occurred in addition to histiocyes, some of which had a crescentic appearance and immunoreactivity for myeloperoxidase. In the residual surrounding parenchyma, small foci of CD303-positive plasmacytoid dendritic cells were observed.

C: The patient fulfills four (i.e., arthritis, ANA positivity, immune alterations, and hematologic involvement) out of the eleven 1997 ACR criteria required for the classification of SLE and can be therefore likely said to be affected by SLE. Moreover the histo-pathologic findings are consistent with KFD/SLE. An aggressive immunosuppressive therapy is now required to induce disease remission.

D: The patient was started on $75 \mathrm{mg} /$ day of prednisone, with prompt benefit; moreover, a gastric and bone protection with pantoprazole, alendronate and calcium plus vitamin D supplementation were also prescribed.

C: High-dose glucocorticoid therapy proved its efficacy in inducing remission of KFD with strong systemic inflammation ${ }^{[1]}$, including cases of KFD-SLE overlap ${ }^{[5]}$. Such dosage prompts patients to a higher risk of stress-ulcer and glucocorticoid-induced osteoporosis; an adequate support therapy is therefore mandatory.

\section{Discussion}

After a struggling diagnostic work-up a diagnosis of KFD was reached by histopathology. Typical histopathologic findings of KFD include: a) the abundance of CD68-positive histiocytes and of plasmacytoid dendritic cells with the absence of neutrophils in the nodal infiltrate; b) irregular necrotic foci within the paracortical regions, around which the histiocytic cells accumulate; c) extensive histiocytic and lymphocytic karyorrhexis within the necrotic areas; d) predominance of CD8-positive T-cells over CD4-positive T-cells and B-cells ${ }^{[1-2]}$. These features point toward signs of cell death associated with increased macrophagic/monocytic activity in KFD. High rates of cell death, persistence of cell-derived debris and activation of monocytic cells within inflamed tissues constitute an ideal setting for the development of autoimmunity ${ }^{[11-12]}$ and are all hallmarks of SLE ${ }^{[11,13]}$. This autoimmune condition, besides sharing many histopathological findings with KFD, also shows several KFD-associated clinical and laboratory findings, such as erythrocyte sedimentation rate, lactate dehydrogenase and transaminase elevations and leukopenia ${ }^{[1,14]}$. Notably, all these features were present in this patient.

Several studies reporting an association between KFD and SLE ${ }^{[3,5,14-15]}$ support the inclusion of KFD as part of the clinical spectrum of SLE. At a variance with this view, other Authors underline the clinicopathologic peculiarity of KFD and support the importance of keeping these two entities separate ${ }^{[1-2,16]}$. In the herein reported patient the association of lymph node enlargement and a limited spectrum of rheumatologic signs (arthritis, leukopenia, Raynaud's phenomenon, and positive autoimmune serology) favored the diagnosis of KFD. Moreover, in contrast with other reports of KFD which 
were excluding SLE ${ }^{[16-17]}$, in the present case the clinical and, mostly, serological signs of an active SLE were markedly evident. Interestingly such clinical presentation (with marked signs of inflammatory activity) and the presence of a diffuse pattern of lymph node involvement seem to be almost invariably reported in SLE-KFD associations ${ }^{[5,18]}$.

In their metanalysis of 244 cases of KFD Kucukardali et al. ${ }^{[3]}$ take also into account the clinical overlap between KFD and adult Still's disease, suggesting possible future investigations about the relationships between these two diseases. Interestingly in the present case, some characteristic features of adult Still's disease (i.e, lymphadenopathy, fever, arthritis, hyperferritinemia, anemia, increased ESR and liver dysfunction) were present, and the potential occurrence of adult Still's disease was indeed hypothesized early in the course of the patient's history.

The early activation of some intra-glandular parotid lymph-nodes (a rare sign of disease onset in $\mathrm{KFD}^{[4]}$ ) had also initially raised the suspicion of Sjögren's syndrome. Notably, while a clinical overlap between SLE and Sjögren's syndrome is quite frequent, especially in young patients, less is known about possible clinical associations between KFD and Sjögren's syndrome.

The diagnosis of KFD is usually achieved by exclusion of many other systemic diseases ${ }^{[2]}$. This task should invariably involve histopathological analysis on lymphoadenectomy, although much effort has been spent to identify reliable imaging (by means of PET/CT scan ${ }^{[19]}$ or ultrasonography ${ }^{[20]}$ ) or laboratory ${ }^{[14]}$ specific markers of KFD.

In this case report we described a paradigm of the polymorphic clinical presentation of KFD, showing in particular how its wide overlap with other immune-mediated disorders could make its diagnosis extremely challenging for the clinician.

\section{Acknowledgements}

We thank Dr. Stefano Franchini for the initial diagnostic work-up of this patient.

\section{References}

[1] Bosch, X. and A. Guilabert, Kikuchi-Fujimoto disease. Orphanet J Rare Dis. 2006; 1: 18. PMid:16722618 http://dx.doi.org/10.1186/1750-1172-1-18

[2] Hutchinson, C.B. and E. Wang, Kikuchi-Fujimoto disease. Arch Pathol Lab Med. 2010; 134(2): 289-93. PMid:20121621

[3] Kucukardali, Y., et al., Kikuchi-Fujimoto Disease: analysis of 244 cases. Clin Rheumatol. 2007; 26(1): 50-4. PMid:16538388 http://dx.doi.org/10.1007/s10067-006-0230-5

[4] Laccourreye, O., et al., Intraparotid subacute necrotizing histiocytic lymphadenitis (Kikuchi-Fujimoto's disease). J Otolaryngol. 1997; 26(1): 64-6.

[5] Kim, S.K., et al., Histiocytic necrotizing lymphadenitis in the context of systemic lupus erythematosus (SLE): Is histiocytic necrotizing lymphadenitis in SLE associated with skin lesions? Lupus. 2011; 20(8): 809-19. http://dx.doi.org/10.1177/0961203310397684

[6] Fox, R.I., Sjogren's syndrome. Lancet, 2005; 366(9482): 321-31. http://dx.doi.org/10.1016/S0140-6736(05)66990-5

[7] Venables, P.J., Sjogren's syndrome. Best Pract Res Clin Rheumatol. 2004; 18(3): 313-29. http://dx.doi.org/10.1016/S1521-6942(04)00036-1

[8] Gaitonde, S., D. Samols, and I. Kushner, C-reactive protein and systemic lupus erythematosus. Arthritis Rheum. 2008; 59(12): 1814-20. http://dx.doi.org/10.1002/art.24316

[9] Fazzini, F., et al., PTX3 in small-vessel vasculitides: an independent indicator of disease activity produced at sites of inflammation. Arthritis Rheum. 2001; 44(12): 2841-50. http://dx.doi.org/10.1002/1529-0131(200112)44:12<2841::AID-ART472>3.0.CO;2-6

[10] Kojima, M., et al., Reactive follicular hyperplasia in the lymph node lesions from systemic lupus erythematosus patients: a clinicopathological and immunohistological study of 21 cases. Pathol Int. 2000; 50(4): 304-12. PMid:10849316 http://dx.doi.org/10.1046/j.1440-1827.2000.01052.x

[11] Munoz, L.E., et al., Autoimmunity and chronic inflammation - two clearance-related steps in the etiopathogenesis of SLE. Autoimmun Rev. 2010; 10(1): 38-42. PMid:20817127 http://dx.doi.org/10.1016/j.autrev.2010.08.015 
[12] Frisoni, L., et al., Nuclear autoantigen translocation and autoantibody opsonization lead to increased dendritic cell phagocytosis and presentation of nuclear antigens: a novel pathogenic pathway for autoimmunity? J Immunol. 2005; 175(4): $2692-701$. PMid:16081846

[13] Blanco, P., et al., Induction of dendritic cell differentiation by IFN-alpha in systemic lupus erythematosus. Science. 2001; 294(5546): 1540-3. PMid:11711679 http://dx.doi.org/10.1126/science.1064890

[14] Cheng, C.Y., et al., Clinical presentations, laboratory results and outcomes of patients with Kikuchi's disease: emphasis on the association between recurrent Kikuchi's disease and autoimmune diseases. J Microbiol Immunol Infect. 2010; 43(5): 366-71. http://dx.doi.org/10.1016/S1684-1182(10)60058-8

[15] Gordon, J.K., et al., Overlap between systemic lupus erythematosus and Kikuchi Fujimoto disease: a clinical pathology conference held by the Department of Rheumatology at Hospital for Special Surgery. HSS J. 2009; 5(2): 169-77. http://dx.doi.org/10.1007/s11420-009-9123-x

[16] Yilmaz, M., et al., Histiocytic necrotizing lymphadenitis (Kikuchi-Fujimoto's disease) mimicking systemic lupus erythematosus: a review of two cases. Lupus. 2006; 15(6): 384-7. PMid:16830886 http://dx.doi.org/10.1191/0961203306lu2320cr

[17] Sousa Ade, A., et al., Kikuchi-Fujimoto disease: three case reports. Sao Paulo Med J, 2010; 128(4): 232-5. PMid:21120436

[18] Cramer, J., et al., Necrotizing lymphadenitis: Kikuchi--Fujimoto disease alias lupus lymphadenitis? Lupus. 2010 ; 19(1): 89-92. PMid:19933723 http://dx.doi.org/10.1177/0961203309345793

[19] Tsujikawa, T., et al., Kikuchi-Fujimoto disease: PET/CT assessment of a rare cause of cervical lymphadenopathy. Clin Nucl Med. 2011; 36(8): 661-4. PMid:21716016 http://dx.doi.org/10.1097/RLU.0b013e31821a2878

[20] Lo, W.C., et al., Ultrasonographic differentiation between Kikuchi's disease and lymphoma in patients with cervical lymphadenopathy. Eur J Radiol. 2011. PMid:21546181 http://dx.doi.org/10.1016/j.ejrad.2011.04.030 